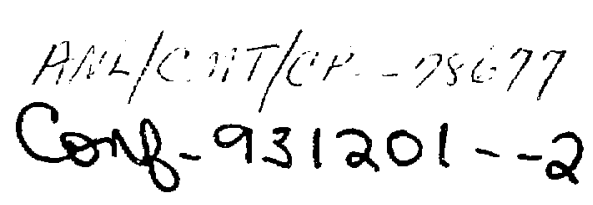

CHARACTERISTICS OF COLLOIDS GENERATED DURING THE CORROSION OF

NUCLEAR WASTE GLASSES IN GROUNDWATER

\author{
X. Feng, E.C. Buck, C. Mertz, J.K. Bates, J.C. Cunnane, D. Chaiko
}

Argonne National Laboratory, Argonne, IL 60439

Chemical Technology Division
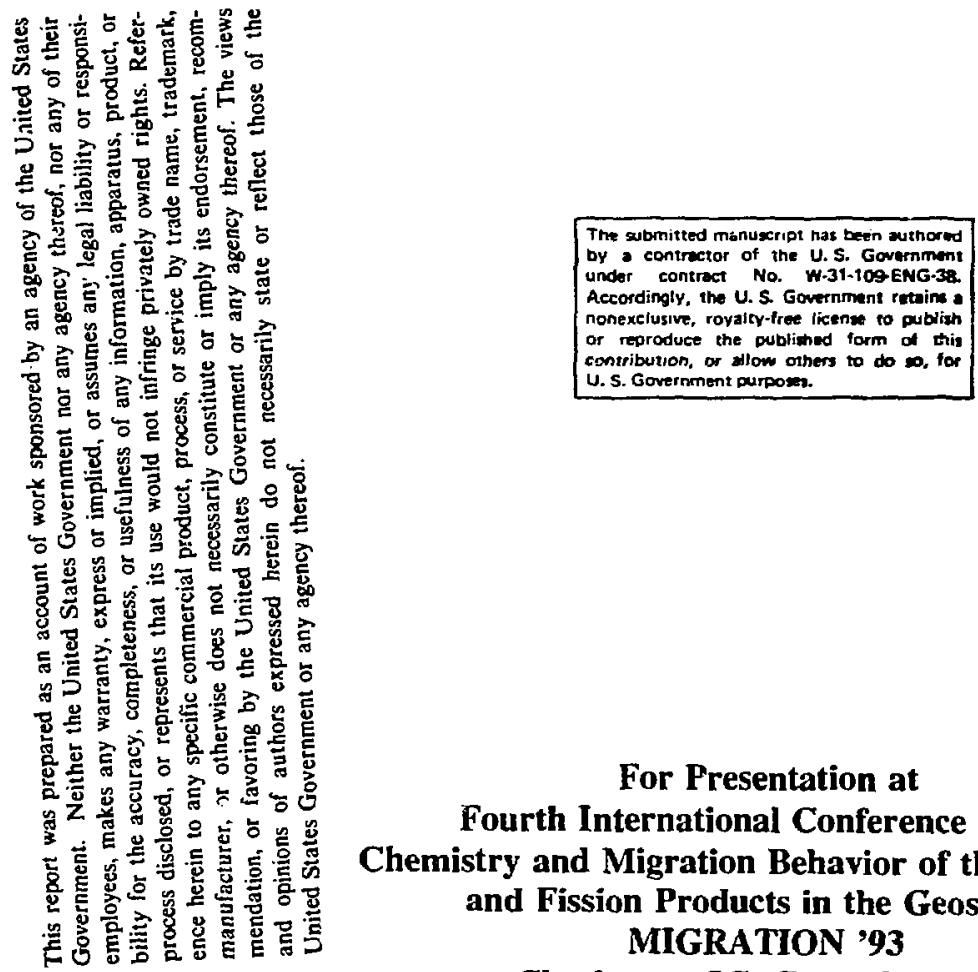

For Presentation at

Fourth International Conference on the

Chemistry and Migration Behavior of the Actinides

and Fission Products in the Geosphere

MIGRATION' 93

Charleston, SC., December 1993

and

Publication in Radiochimica Acta

October 1993 


\section{ChARACTISTICS OF COLLOIDS GENERATED DURING THE CORROSION OF NUCLEAR WASTE GLASSES IN GROUNDWATER}

X. Feng, E. C. Buck, C. Mertz, J. K. Bates, J. C. Cunnane, and D. Chaiko

Argonne National Laboratory, Chemical Technology Division, Argonne, IL 60439

\section{ABSTRACT}

Aqueous colloidal suspensions were generated by reacting nuclear waste glasses with groundwater at $90^{\circ} \mathrm{C}$ at different ratios of the glass surface area to solution volume (S/V). The colloids have been characterized in terms of size, charge, identity, and stability with respect to salt concentration, $\mathrm{pH}$, and time, by examination using dynamic light scattering, electrophoretic mobility, and transmission electron microscopy. The colloids are predominately produced by precipitation from solution, possibly with contribution from reacted layers that have spallated from the glass. These colloids are silicon-rich minerals, such as smectites and uranium silicates. The colloidal suspensions agglomerate when the salinity of the solutions increase. The agglomerated particles can be resuspended if the salt concentration is lowered by dilution. The colloids agglomerate quickly when the leachate is cooled down to room temperature and settle out of the solution within a few days. The colloids have an isoelectrical point at a pH of approximately 1 . Between $\mathrm{pH} 1$ and 10.45, the colloids are negatively charged. The average colloid size is largest at the isoelectrical point and is smallest around $\mathrm{pH} 6$, is related to the measured zeta potentials of the colloids.

The following implications for modeling the colloidal transport of contaminants have been derived from this study: (1) The sources of the colloids are not only solubility-limited real colloids and the pseudo colloids formed by adsorption of radionuclides onto a groundwater colloid, but also from the spalled surface layers of reacted waste glasses. (2) In a repository, the local environment is likely to be glass-reaction dominated and the salt concentration is likely to be high, leading to rapid colloid agglomeration and settling; thus, colloid transport may be insignificant. (3) If large volumes of groundwater contact the glass reaction site, the precipitated colloids may become resuspended, and colloid transport may become 
important;. (4) Under most conditions, the colloids are negatively charged and will deposit readily on positively charged surfaces. Negatively charged surfaces will, in general, facilitate colloid stability and transport.

\section{INTRODUCTION}

Colloids have been recognized to be important in the transport of radionuclides and other hazardous contaminants in a waste repository. Most of the colloidal studies [ $\hat{i}-4]$, however, have concentrated on those already present in groundwater. These studies have focused primarily on two types of colloids: real colloids that are formed by solubility-limited hydrolysis and polymerization of actinides, and pseudo colloids that are formed by adsorption of radionuclides onto existing groundwater colloids. The formation of suspended material and colloids due to the reaction of waste glass and its implications for nuclear waste management have only recently been considered to play a role in the long term behavior of glass [5-7]. These studies have indicated that during the corrosion of waste glass in groundwater, the metastable glass may be transformed into a more stable assemblage that may spall from the reacted layer to become a new source of colloidal material and is called primary colloids [5]. The colloids formed in such a system displayed a wide size distribution ranging from $3.8 \mathrm{~nm}$ to $>1.0 \mu \mathrm{m}$, based on serial filtration results [8]. Kawano and Tomita [9] observed the formation of allophane and beidellite during the laboratory hydrothermal alteration of volcanic glass, and Buck et al.[10] have shown that smectite clays may nucleate in solution during the reaction of nuclear waste glass in immersion tests. The processes which cause the formation of colloids during waste glass reaction have been defined in terms of condensation (spallation) and precipitation (nucleation) reactions by Ahn et al. [11] Whether one dominates over the other depends on the test conditions,. There is evidence for both processes occurring [12], although.

Colloids are commonly described as particles between $1 \mathrm{~nm}$ and $1 \mu \mathrm{m}$ in diameter, however an alternate definition is particles capable of remaining suspended in an undisturbed aqueous solution for an indefinite period. The colloids discussed in this paper are defined as 
particles, regardless size, that can form a stable (on the time scale of our experiments) aqueous suspension.

The objective of this study is to characterize the colloids formed during waste glass corrosion under repository-relevant conditions, and to show that the information obtained is pertirent to (1) resolving issues concerning the contribution of colloids to the source term for radionuclide release and (2) providing a data base for potential engineered barrier systems to prevent colloidal transport of radionuclides. This report contains results of cur studies on (1) colloid generation, (2) the stability of colloidal suspensions, (3) the effects of $\mathrm{pH}$ on the particle size distribution and charge (zeta potential), and (4) the source and nature of the colloids.

\section{EXPERIMENTAL}

\section{Method for Colloid Generation}

The colloids were generated through corrosion of either SRL131S glass or actinide-doped SRL131A glass in $\mathrm{J}-13$ well water at $90^{\circ} \mathrm{C}$. These glass compositions were developed for the immobilization of the defense high-level nuclear wastes at the Westinghouse Savannah River site. The EJ-13 water is a representative groundwater expected at the potential highlevel nuclear waste disposal site, Yucca Mountain. The Glass and EJ13 compositions are provided elsewhere [13].

The test procedure used is a modified PCT method [14]. In each test, the requisite quantity of 100- to 200- mesh powder glass was immersed in a volume (depending on the desired ratio of surface area of glass to solution volume (SAV)) of $J-13$ well water in a Teflon reaction vessel. The test vessel was tightly sealed with the cap and placed in a $90^{\circ} \mathrm{C}$ oven. The vessels were periodically checked to ensure that the vessels were not leaking. After a test was terminated, aliquots of the glass leachate were removed and analyzed for colloids, $\mathrm{pH}$, cation, and anion concentrations. This type of test is a static test in which the leachate is undisturbed during the test period and may be representative of a glass canister which is 
filled with water but where little fluid exchange occurs.

\section{Size measurements}

The colloids were measured with a dynamic laser light scattering system, a Malvem Instruments System $4700 \mathrm{C}$ photon correlation spectrometer with a $35-\mathrm{mW}$ laser as the light source. The autocorrelation function was measured at $25^{\circ} \mathrm{C}$ and at a scattering angle of $90^{\circ}$. A 64-channel correlator was used in the parallel (geometrically spaced channels) and serial (equally spaced channels) modes. Most of the data was collected in the serial mode.

During a measurement the fluctuation in scattering intensity from a sample is recorded for a time period of a few nanoseconds to several seconds. The fluctuations which arise from the diffusion of particles through the laser beam depends on the diffusion rate, the wavelength of the laser, and the angle of observation. The intensity and fluctuations of the scattered light are measured as discrete photons by a photomultiplier. The output from the photomultiplier is fed into a correlator that calculates the autocorrelation function. From this function, a diffusion coefficient can be obtained, then, a particle size can be estimated through the well-known Stokes-Esinstein equation [15].

The correlation function was analyzed using CONTIN [16-18], a model independent data analyzing program that inverts the time correlation function to give a distribution of relaxation times. The colloidal suspension generated from the interaction of glass with groundwater is polydisperse. It is usually characterized by a single smooth and broad peak, but it may produce multiple jagged peaks, in the CONTIN size distribution plots. The former was usually chosen in our data analyses to derive the average particle size as a function of $\mathrm{pH}$ and time. Thus, our emphasis was on the relative particle size, not on the absolute size values.

\section{Zeta potential measurements}

The Pen Kem LASER ZEE ${ }^{\text {tm }}$ MODEL 500 was used to measure the electrophoretic mobility in terms of zeta potential. These measurements were made immediately after the $\mathrm{pH}$ of the 
solution was adjusted to the desired value by addition of ultrapure $\mathrm{HNO}_{3}$ or $\mathrm{NaOH}$ solution. Usually, four to six measurements were performed on each test solution to derive an average zeta potential. The solution $\mathrm{pH}$ usually drifted to a slightly lower value during zeta potential measurements. The averages of the zeta potential values were used for data analysis, since some particles (fewer than 10\%) were observed to have the opposite charge of most of the colloids during the measurements. This observation is probably a characteristic of the waste glass colloidal system, because nuclear waste glass usually contains more than 20 elements and the groundwater also has a complex composition.

Transmission Electron Microscopy (TEM) examinations

A small quantity of unfiltered leachate was extracted shortly after the waste glass test was terminated and a drop of the leachate was wicked through a 'holey' carbon grid, which acted as a filter $[8,10]$. This technique caused the colloidal particles to agglomerate and may have affected the oxidation states. Controls were run to determine possible particulate contamination routes. Evaporities were sometimes found to precipitate on the grid, from evaporation of small droplets of leachate remaining on the surface of the carbon film.

Contaminants on the 'as-received' grids were usually easily recognized in the microscope as being large particles, often single crystal, of materials such as graphite. Analyses were performed using a JEOL 2000FXII transmission electron microscope operated at $200 \mathrm{kV}$ and equipped with two energy dispersive $\mathrm{x}$-ray spectrometers. Analysis of phases involved the use of the energy dispersive $x$-ray spectroscopy (EDS) and selected area electron diffraction

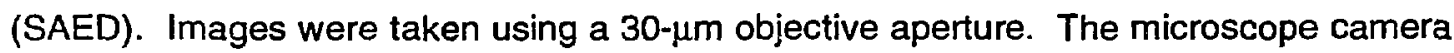
lengths were calibrated using a polycrystalline aluminum standard.

\section{RESULTS}

\section{(1) Colloid Generation}

Our first concern was to generate colloidal solution with enough volume for this study. The first four tests (designated $A-1, A-2, A-3$, and $A-4$ ) were carried out with SRL 131S glass and at an SA/V of $11,400 \mathrm{~m}^{-1}$. After 80 days at $90^{\circ} \mathrm{C}$, the four test vessels were removed from 
the oven, and about $1 \mathrm{~mL}$ of leachate was carefully withdrawn from each vessel to check for colloids using the laser light scattering system. The results of several measurements of the leachates indicated that the concentrations of the colloids were so low that the counting rates, counts per second (CPS), of the light scattering measurements were close to the background level (i.e., similar to the CPS of the starting $J-13$ well water). This indicates that virtually no additional colloids were produced by glass reaction.

In earlier static testing of SRL $202 \mathrm{U}$ glass in J-13 well water [10], the amount of colloids in the leachate first increased steadily and then decreased with time. The time it takes for the initial colloid increase to drop to a very low concentration depends on the SAV of the tests. These durations were 300,200 , and 100 days for SAV of 340,2000 , and $20000 \mathrm{~m}^{-1}$, respectively. The very low concentration of colloids observed for SRL 131S at an SAN of $11400 \mathrm{~m}^{-1}$ is therefore consistent with the observations for SRL 202U at an SAN of 20000 $\mathrm{m}^{-1}$.

To better understand the above effect, a dilution experiment consisting of four tests (A-1-1, $A-1-2, A-1-3$, and $A-1-0$ ) was performed. The tests used the leachate, reacted glass powders, precipitates from the terminated test, $A-1$, and fresh $\mathrm{J}-13$ water. Table I shows the test parameters.

Table 1. Parameters for the Dilution Tests

\begin{tabular}{|l|l|l|l|l|}
\hline & $\mathrm{A}-1-1$ & $\mathrm{~A}-1-2$ & $\mathrm{~A}-1-3$ & $\mathrm{~A}-1-0$ \\
\hline Leachate (m) & 1.0 & 2.0 & 3.0 & $25^{*}$ \\
\hline Reacted Glass and Precipitates $(\mathrm{g})$ & 1 & 1 & 1 & 17 \\
\hline Fresh $\mathrm{J}-13$ Water (ml) for dilution & 32.0 & 31.0 & 30.0 & 10.0 \\
\hline
\end{tabular}

* This is the leftover solution from test A-1 after removing leachate for size measurements and for the new tests $(A-1-1, A-1-2$, and $A-1-3)$.

These dilution tests were carried out at $90^{\circ} \mathrm{C}$ for 4 days. At the end of the tests, $\sim 1 \mathrm{~mL}$ of solution was withdrawn from each vessel. The CPS values of the laser scattering measurements were $2,000,11000,10500$, and 12000 for Tests $A-1, A-1-1, A-1-2$, and A- 
1-3, respectively. Thus, the CPS increased about 5-6 times over the original A-1 solution; however, the CPS for Test A-1-0 increased by only a factor of two (to 4000 ). These values indicate that there are sufficient colloids in A-1-1, A-1-2, and A-1-3 for size and zeta potential measurements, but not in $A-1-0$ or $A-1$. In a separate dilution test similar to $A-1-3$, but which was equilibrated for 5 days, the CPS value measured was 60,000 , which is a factor of 30 increase over that of $A-1$ solution.

To prepare enough colloidal solution for our subsequent studies, all the solutions withdrawn from $A-1-1, A-1-2$, and $A-1-3$ were combined and the resulting colloidal solution was designated A-1-ALL. Its CPS value was 10500 .

Two tests, $\mathrm{B}-1$ and $\mathrm{B}-2$, were carried out with SRL $131 \mathrm{~A}$ glass powders in $\mathrm{J}-13$ well water at $90^{\circ} \mathrm{C}$ for 560 days at an SAN of $2000 \mathrm{~m}^{-1}$. At the temination of these tests, the CPS values of the leachates were measured to be $\sim 22000$, which is suitable for this study.

\section{(2) Stability of colloidal suspension vs. time}

The stability of $\mathrm{B}-1$ and $\mathrm{B}-2$ colloidal solution was monitored at room temperature by determining the sizes and the amounts of colloids versus time. The leachates were transferred directly from the test vessel into a vial for light scattering measurements. The first measurement at room temperature was performed about 1 hour (0.04 days) after the test was terminated. The mean colloidal particle size from $B-1$ versus time plot is shown in Fig. 1a. The data indicate that the colloids began to agglomerate when the leachate was at room temperature. The mean particle size increased to about $1250 \mathrm{~nm} 1.25$ days after the test termination. Then these large colloids began to settle out of solution, and after five days only small colloids remained in solution. When the measuring vial was shaken, the large agglomerates were resuspended in solution, but they quickly settled out again, as shown in Fig. 1a. The mean colloid size measured before shaking was nearly the same as that one hour after shaking, indicating that only the small colloids, those $-600-700 \mathrm{~nm}$, remained suspended in solution. 
The CPS value of $\mathrm{B}-1$ solution, shown in Fig. 1b, is a measure of the amount of the colloids, that depends on their number and sizes. The data in Fig. $1 \mathrm{~b}$ display the same trend as that in Fig. 1a. The CPS value increases as the colloids become larger and decreases as the colloids settle out of solution. The CPS values decrease from the initial value of about 22000 to about 2000 , which is the same value as the blank $J-13$ well v/ater. Thus, most of the colloids generated during glass corrosion settled out of the solution after about five days at room temperature. The colloid stability in the B-2 solution is in general agreement with the results of the B-1 solution, with most of the colloids in B-2 settling out of solution in about five days.

\section{(3) Zeta potential and size at different pH}

The effects of solution $\mathrm{pH}$ on zeta potential and particle size were studied using $60 \mathrm{~mL}$ of the A-1-ALL solution. The original A-1-ALL solution had a $\mathrm{pH}$ value of 9.14 , a zeta potential of $-16 \mathrm{mV}$, and a mean particle size of $638 \mathrm{~nm}$. Then ultrapure nitric acid was added to half of this solution, which solution was labeled as A-1-ALL-D, to lower the solution $\mathrm{pH}$ downward. The $\mathrm{pH}$ of the rest solution (A-1-ALL-U) was increased by adding $1 \mathrm{M}$ or $10 \mathrm{M} \mathrm{NaOH}$ solution.

The measured zeta potentials of the leachate over the $\mathrm{pH}$ range of 1 to 10.5 are presented in Table II and plotted in Fig. 2a.

Table II. Zeta Potential (Z.P.) Measurements on A-1-ALL

\begin{tabular}{|l|l|l|l|l|l|l|l|l|l|l|}
\hline $\mathrm{pH}$ & $\mathrm{pH}$ & $\mathrm{pH}$ & \multicolumn{3}{|l|}{ Z. P. Measurements, mV } & Z.P. mV & $\begin{array}{l}\text { StD. } \\
\text { Dev }\end{array}$ \\
\hline (before) & (after) & (mean) & 1 & 2 & 3 & 4 & 5 & 6 & (mean) & \\
\hline 1.06 & 1.01 & 1.04 & 0 & 1 & 2 & 0 & ND & ND & 0 & 1 \\
\hline 2.77 & 2.66 & 2.72 & -36 & -40 & -42 & -46 & ND & ND & -41 & 4 \\
\hline 3.99 & 3.93 & 3.96 & -34 & -44 & -50 & -60 & -35 & -42 & -44 & 9 \\
\hline 6.33 & 6.07 & 6.20 & -72 & -65 & -60 & -66 & ND & ND & -66 & 4 \\
\hline 7.48 & 7.26 & 7.37 & -23 & -24 & -25 & -26 & ND & ND & -25 & 1 \\
\hline 9.14 & 9.01 & 9.08 & -18 & -19 & -11 & -17 & -16 & -13 & -16 & 3 \\
\hline 10.50 & 10.40 & 10.45 & -44 & -41 & -42 & -45 & ND & ND & -43 & 2 \\
\hline
\end{tabular}

ND $=$ Not Done

As shown in Table II, the solution pH usually drifted to a slightly lower value during the zeta potential measurements. The isoelectric point where the zeta potential is zero, as shown in 
Fig. 2a, was at a pH of about 1 . The colloidal particles were always negatively charged when the $\mathrm{pH}$ of the solution was above 1 .

The size of the colloids was expected to correlate closely with solution $\mathrm{pH}$ and zeta potential. The particle size in the A-1-ALL solution was measured at each $\mathrm{pH}$ after the corresponding zeta potential measurement. Figure $2 \mathrm{~b}$ shows the measured size variations with solution $\mathrm{pH}$. The particle size is largest at the isoelectric point $(\mathrm{pH} 1)$, second largest at $\mathrm{pH}$ of 9.14 , and smallest around $\mathrm{pH} 6$. These trends are similar to those observed for the zeta potential curves(Fig. 2a).

\section{(4) TEM examinations}

Colloidal particles were characterized by TEM. The analysis on the B-2 solution was performed after the stability-vs.-time study described above. The B-2 solution was, therefore, shaken to resuspend the precipitated materials before being sampled for TEM analyses. Several different types of particles were observed during TEM analyses. Smectite was present, which exhibited the characteristic turbostratic oblique textured electron diffraction (OTED) pattern, that is typical of smectite clay minerals [19], as seen in Fig. 3.

The clay particles were not well-textured but showed signs of edge-face agglomeration, which resulted in an only slightly elliptica! OTED pattern (Figs. 3a and 3b). Large needle-like crystals sometimes exceeding $3 \mu \mathrm{m}$ in length, of a uranium silicate were also found. Electron diffraction and EDS compositional analysis were used to identify the phase as weeksite (Fig. 4). The weeksite suspended material was often found associated with particles of clay, as seen in Fig. 4b. The same weeksite phase was also observed in the surface layers of the reacted glass in the B-2 test, as shown in Fig. $4 a$, suggesting that the material present in the leachate had spalled off the reacted layer.

Two other colloidal solutions were examined with TEM: A-1-ALL-U, which was produced by increasing the $\mathrm{pH}$ of $\mathrm{A}-1-\mathrm{ALL}$ to 10.5 and $\mathrm{A}-1-\mathrm{ALL}-\mathrm{D}$, which was produced by decreasing the $\mathrm{pH}$ of $\mathrm{A}-1-\mathrm{ALL}$ to 1.0. The TEM analyses were performed one week after these two 
solutions were used in the particle size-zeta potential-pH study. Thus, these solutions had been at pHs of 1.0 and 10.5, respectively, for more than one week. The small droplets of the colloidal solution used in TEM analysis were taken from unshaken colloidal solutions. The A1-ALL-D sample had virtually no colloidal particles present, while the A-1-ALL-U sample contained a large amount of colloidal matter, including smectite clay and a calcium silicon phosphate phase, identified as nagelschmidtite. A similar phosphate phase has also been located in the surface layers of reacted SRL 202U glass [20].

\section{DISCUSSION}

\section{(1) Colloid stability is a function of salt concentration}

It is well known that the addition of electrolytes to a colloidal system can compress the diffuse counter-ion atmosphere (electric double layer) toward the surface of the colloids, causing the colloidal particles to agglomerate by van der Waals attraction and to eventually settle out of solution. The time period during which the colloid concentrations of SRL 202U leachates initially increased and then decreased was observed to shorten as the SAV changed from 340 to $20000 \mathrm{~m}^{-1}$ [10]. Tests conducted at higher SAV result in more glass reaction and higher salt concentrations in the leachate because more glass surface area is available for glass reaction in the limited solution volume. The salt concentration in higher SAN tests, then, reaches the concentration level that can flocculate a colloidal suspension sooner than in the lower SAN tests. It is likely that the high concentration of salts produced in the 80-day leachate of SRL 131S at SAN of $11400 \mathrm{~m}^{-1}$ (samples A-1, A-2, f-3, and A-4) had already caused most of the colloids to flocculate, which resulted in the low CPS values measured by light scattering.

The dilution experiments verified this salt effect. In these tests, the colloid solution was diluted with fresh $\mathrm{J}-13$ well water and was equilibrated with the precipitated colloids for several days at $90^{\circ} \mathrm{C}$. When the salt concentration was lowered by this dilution, compression of the double layer of the colloids was reduced. The expanded double layer decreased the vai der Waals attraction and increased the repulsive force between the colloidal particles, resulting in the 
resuspension of the flocculated colloids into solution. This was evidenced when the CPS values increased by as much as a factor of 30 of the leachates in the dilution experiment.

This salt effect is important because a repository setting is likely to be in a nearly static condition with minimal groundwater in contact with the glass. Glass corrosion in such a situation will likely produce a leachate with a high salt content and a high $\mathrm{pH}$. Under these conditions, the colloid concentration will probably be minimal, as will the colloidal transport of radionuclides. On the other hand, if a large amount of groundwater with a low' salt content contacts the glass reaction site, the precipitated colloids may become resuspended. Alternatively, if the groundwater has a high salt content (such as in a salt repository), the formation of colloids may be prohibited.

\section{(2) Charge and size of colloids are a function of $\mathrm{pH}$}

The $\mathrm{pH}$ range of the leachate resulting from waste glass corrosion in groundwater, especially under static conditions, varies from near 7 to 13 , as glass composition and SAN change [22]. The charge of the colloids strongly depends on leachate $\mathrm{pH}$. As a result, the charge at a particular $\mathrm{pH}$ is important in understanding the colloid behavior. It will determine what surfaces (positive or negative) will attract the colloids. The particle size of the colloids also changes with solution $\mathrm{pH}$. At the isoelectric point, the net charge of the colloids becomes zero, and the colloids agglomerate quickly. The charge and size of the colloids may have a very large influence on their stability, transport properties, and interactions with the glass surface, canister wall, and packing materials.

The mineral compositions of the colloids influence their zeta potentials, which also vary significantly with solution $\mathrm{pH}$. The TEM examinations of these waste glass colloids revealed mainly Si-rich phases, such as smectites and uranium silicates. The richness in silica makes these colloids similar to a pure silica colloidal system.

The graph of stability versus $\mathrm{pH}$ of a pure silica colloidal system (Fig. 5) exhibits similar trends seen in Fig. 2. When the solution pH increases from 1 to 6 , the negative charge on the 
colloid increases rapidly, probably due to deprotonation from $\mathrm{Si}-\mathrm{O}-\mathrm{H}, \mathrm{Al}-\mathrm{O}-\mathrm{H}$, and $\mathrm{M}-\mathrm{O}-\mathrm{H}$ (where $M$ represents metal ions) sites. Between pHs 6 and 9.14, the negatively charged colloids may adsorb some positively charged hydrolyzed cations, such as $\mathrm{M}(\mathrm{III}) \mathrm{OH}^{2+}$ and $\mathrm{M}(I I) \mathrm{OH}^{+}$, because many metal cations can be easily hydrolyzed in this $\mathrm{pH}$ range. This adsorption reduces the negative charge on the colloids. At $\mathrm{pH} 10.5$, most hydrolysis products of metal cations are neutral or negatively charged, the $\mathrm{pH}$ is also close to the $\mathrm{pK}_{2}$ of silicic acid, and the negative charge on the colloids increases again (Fig. 2a). The presence of other elements in the colloids from our experiments causes the observed deviations from the pure silicon colloidal suspension, i.e., the isoelectric point is at $\mathrm{pH} 1$ for the colloids generated by glass corrosion instead of at pH 2 as shown in Fig.5 for pure silica colloids [23].

The size of the colloids was expected to correlate closely with solution $\mathrm{pH}$ and zeta potentials (Fig. 2). In our tests with waste glass colloids, the colloids have zero charge at pH 1 and the particle size increases owing to van der Waals attraction, which draws two particles together at the moment of collision. This attraction produces the largest colloidal size observed for the A-1-ALL solution. At the intermediate $\mathrm{pH}$ (about 6 ), the colloids are negatively charged; they collide much less frequently and less effectively, owing to the increasingly similar charges of the colloids. The smallest colloids were observed at this $\mathrm{pH}$. As the $\mathrm{pH}$ is further increased toward 9.14, the particles are less negatively charged, and they repel each other less effectively than around $\mathrm{pH} 6$, resulting in size growth due to increased collisions. The second largest size was observed at pH 9.14, which is consistent with the region labeled as "particle growth" in Fig.5.

Thus, both particle size and charge contribute to the colloid stability. When the colloids become too large to be suspended in the solution, the colloids settle due to gravity. For instance, the largest mean colloid size for the A-1-ALL solution was observed at $\mathrm{pH} 1$, immediately after $\mathrm{pH}$ adjustment. However, these large colloids (in A-1-ALL-D) settled almost completely out of solution within one week, as shown by light scattering and TEM analyses. When the zeta potential of the colloids is opposite to the zeta potential of the glass 
surface, the colloids may be adsorbed onto the glass surface to form a precipitated surface layer [24]. This may also happen when the two are similarly charged but have low potentials. If the colloids have the same sign as the glass surface and relatively high zeta potential, they may be repelled from the glass surface and grow in the solution [24].

\section{(3) Source of colloids}

Bates et al. [5] have demonstrated that the colloids generated by the interaction of nuclear waste glass with groundwater originated mainly from spalled glass surface layers and from solubility-limited precipitation. The resuits from this study suggest that the waste glass contributes to the colloid formation by leaching, which increases the ion concentration to the level at which nucleation of the colloids, particularly smectite clays occurs. The sudden appearance of the weeksite particles in the leachates at various SAN's and the similar phase observed both in the surface layer (Fig. 4b) of the reacted glass and in the leachates (Fig. 4a) also suggest that the colloids may be formed from fragments of spalled surface layers of the glass. In modeling the colloidal transportation of radionuclides, the sources of the colloids should not only be from solubility-limited precipitation and adsorption of radionuclides onto existing groundwater colloids, but also from spallation of the altered waste glass surface layers. The observation that the colloidal particles are often elongated or planar is also interesting, since most models of colloidal migration assume a spherical geometry for colloids, as do programs used in light scattering to determine size distributions of colloids.

\section{CONCLUSION}

The generation of colloids during corrosion of high-level nuclear waste glasses in a groundwater at $90^{\circ} \mathrm{C}$ has been investigated. The stability of these colloids was determined with respect to the salinity of the solutions, $\mathrm{pH}$, time, particle size, and zeta potential. The composition, morphology, and source of the colloids were also characterized with TEM. The waste glass may contribute to the colloid formation by increasing ion concentrations in the leachate, resulting in nucleation of colloids, and by spalling colloidal size fragments from surface layers of the reacted glass. The colloids are mainly smectite clays and weeksite. High 
concentration of colloids can only be produced when salt concentrations are low; that is they have low SAN ratios (340 and $2000 \mathrm{~m}^{-1}$ ) for relatively long reaction times or high SAV ratios ( 11400 and $20000 \mathrm{~m}^{-1}$ ) for relatively short reaction times. A colloidal suspension can be flocculated if the salt concentration increases. However, the flocculated colloids can be resuspended if the salt concentration is lowered by groundwater dilution. The colloids agglomerate quickly at room temperature. Most of the colloids settle out of the solution at ambient temperature within about five days. The colloids have an isoelectric point at a $\mathrm{pH}$ of about 1 and are negatively charged between $\mathrm{pH} 1$ and 10.5. The size of the colloid particles is largest at the isoelectric point after which it drops and increases again at $\mathrm{pH} 9.14$. The size change observed can be explained by the solution $\mathrm{pH}$ and the zeta potentials.

The implications of the results from this study are as follows. First, in a yiass-reaction dominated repository setting, where salt concentrations are likely to be high when glass reaction occurs, the colloid concentration is likely to be low, and the colloid transport of radionuclides should be minimal. Second, when a large amount of groundwater contacts the glass reaction site, the precipitated colloids may become resuspended, and colloid transport may become important. Third, at ambient temperature, the colloids may agglomerate quickly and settle out of solution in a short time. Fourth, at most pHs (1.0-10.5), the colloids are negatively charged, and any positively charged surface may adsorb or precipitate these colloids. Finally, the sources of the colloids in a waste repository come from both existing colloids in the groundwater and contribution from the waste forms.

\section{ACKNOWLEDGMENTS}

Work supported by the U.S. Department of Energy under Contract W-31-109-ENG-38. The authors thank R. Riel for preparing the manuscript.

\section{REFERENCES}

[1] J. D. F. Ramsey, Radiochimica Acta, 44/45, 165(1988).

[2] E. S. Patera, D.E. Hobart, A. Meijer, R. S. Rundberg, and J. Radioanal. Nucl. Chem. Artic. 
142, 331(1990).

[3] H. Nitsche, Radiochim. Acta 52/53, 3(1991).

[4] J. I. Kim. Radiochim Acta 52/53, 71(1991).

[5] J. K. Bates, J. P. Bradley, A. Teetsov, C. R. Bradley, and M. Buchholtz ten Brink, Science, 256, 649 (1992).

[6] J.I. Kim, W. Treiber, Ch. Lierse and P. Offermann, "Solubility and Colloid Generation of Plutonium From Leaching of A HLW Glass In Salt Solutions," Mat. Res. Soc. Symp. Proc. 44. 359(1985).

[7] C. Degueldre, Mat. Res. Soc. Symp. 294. 817(1993)

[8] J. C. Cunnane and J. K. Bates, Ceram. Trans. 23, 65(1991)

[9] M. Kawano and K. Tomita, Clays and Clay Minerals, 40, 666-674 (1992)

[10] E. C. Buck, J. K. Bates, J. C. Cunnane, W. L. Ebert, X. Feng, and D. J. Wronkiewicz, Mat. Res. Soc. Symp. Proc., 287, 199-206(1993)

[11] T. Ahn, Mat. Res. Soc. Symp. Proc., 287, 1(1993)

[12] X. Feng, E. C. Buck, C. Mertz, J. K. Bates, J. C. Cunnane, and D. J. Chaiko, Proc. Waste Management '93, Feb 28 to Mar 4, Tucson, AZ, vol 2, 1015-1021 (1993)

[13] J. K. Bates et al, ANL Technical Support Program for DOE Environmental Restoration and Waste Management. Annual Report. Oct. 91 - Sept. 92, Argonne Report ANL-93/13, Argonne, IL(1993)

[14] C. M. Jantzen, N. E. Bibler, D.C. Beam, W.R. Ramsey, and B.J. Waters, Westinghouse Savannah River Co. Report, WSRC-TR-90-539, Rev.2, Aiken, SC. (1990).

[15] B. Chu, in Laser light Scattering. Basic Principles and Practice, 2nd Ed. Academic Press, New York, 1991.

[16] S. W. Provencher, Compu. Phys. Commun. 27, 213 (1982).

[17] S. W. Provencher, Compu. Phys. Commun. 27, 229 (1982).

[18] S. S. Sorlie and R. Pecora, Macromolecules $\underline{23}, 487$ (1990).

[19] V. A. Drits, in Electron Diffraction and High Resolution Electron Microscopy of Mineral Structures, Translated by B. B. Smollar, Springer-Verlag, New York, p. 149 (1983). 
[20] W. L. Ebert, J. K. Bates, E. C. Buck, and C. R. Bradley, Mat. Res. Soc. Symp. Proc. Vol. 294. 137(1993).

[21] H. van Olphen, in Clave Colloid Chemistry, and Ed., John Wiley \& Sons, New York (1977).

[22] X. Fend, I.L. Pug, Q. Yam, X. Mao, and P.B. macedo, "Effects of pH on the Leaching Mechanism of Nuclear Waste Glasses," Nuclear Waste Management IV, Ceramic Transactions, 23,G.G. Wicks, D.F. Bickford, and L.R. Bunnell, eds, 95(1991).

[23] R. K. Ier, in The Chemistry of Silica, John Wiley \& Sons, New York (1979).

[24] L. L. Hench and D. E. Clark, Surface Properties and Performance Prediction of Alternative Waste Forms, U.S. Nuclear Regulatory Commission Report, NUREG/CR-3472, (1986).

16 


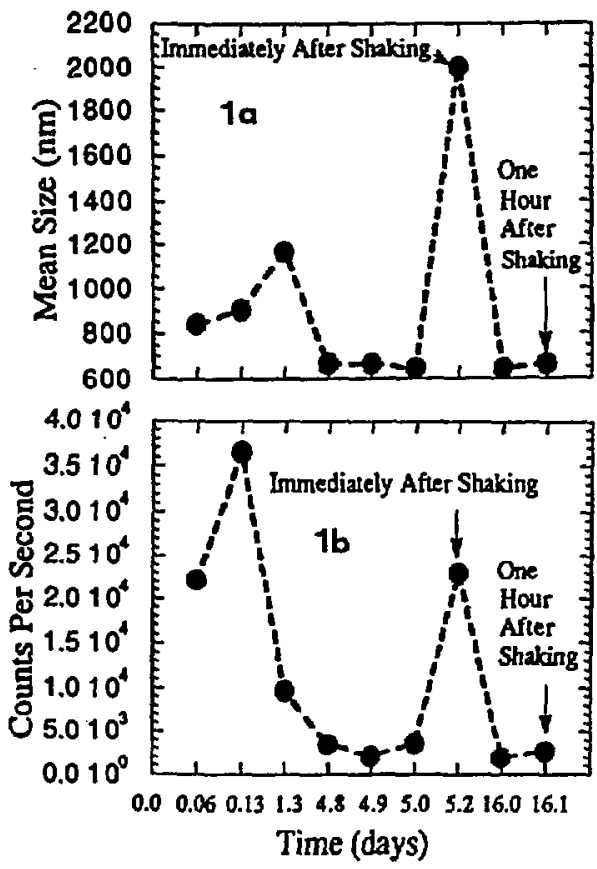

Fig. 1. Results for Test B-1 at Room Temperature. (a) Mean Colloid Size vs. Duration. (b). Colloid Concentration Expressed as Counts Per Second vs. Duration. 


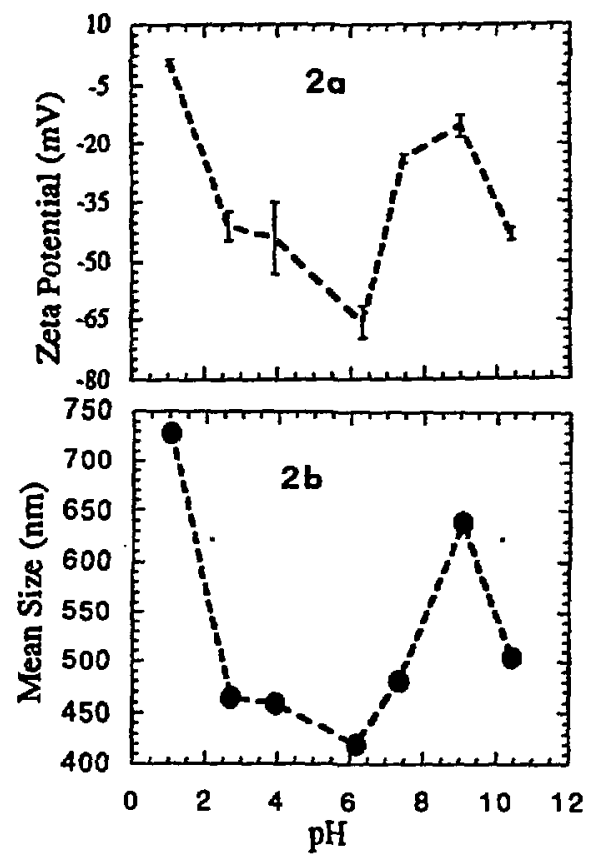

Fig. 2. Results for A-1-ALL Colloidal Solution. (a) Zeta Potential vs. pH. (b) Mean Particle Size vs. pH. 
Fig. 3. TEM Results for A-1-ALL Colloidal Solution. (a) Electron diffraction patterns of smectite clay colloids. taken at $0^{\circ}$ tilt and (b) $35^{\circ}$ tilt show only some signs of texturing. (c) TEM micrograph of the aggiomerated colloids, and (d) EDS compositional analysis of the phase.
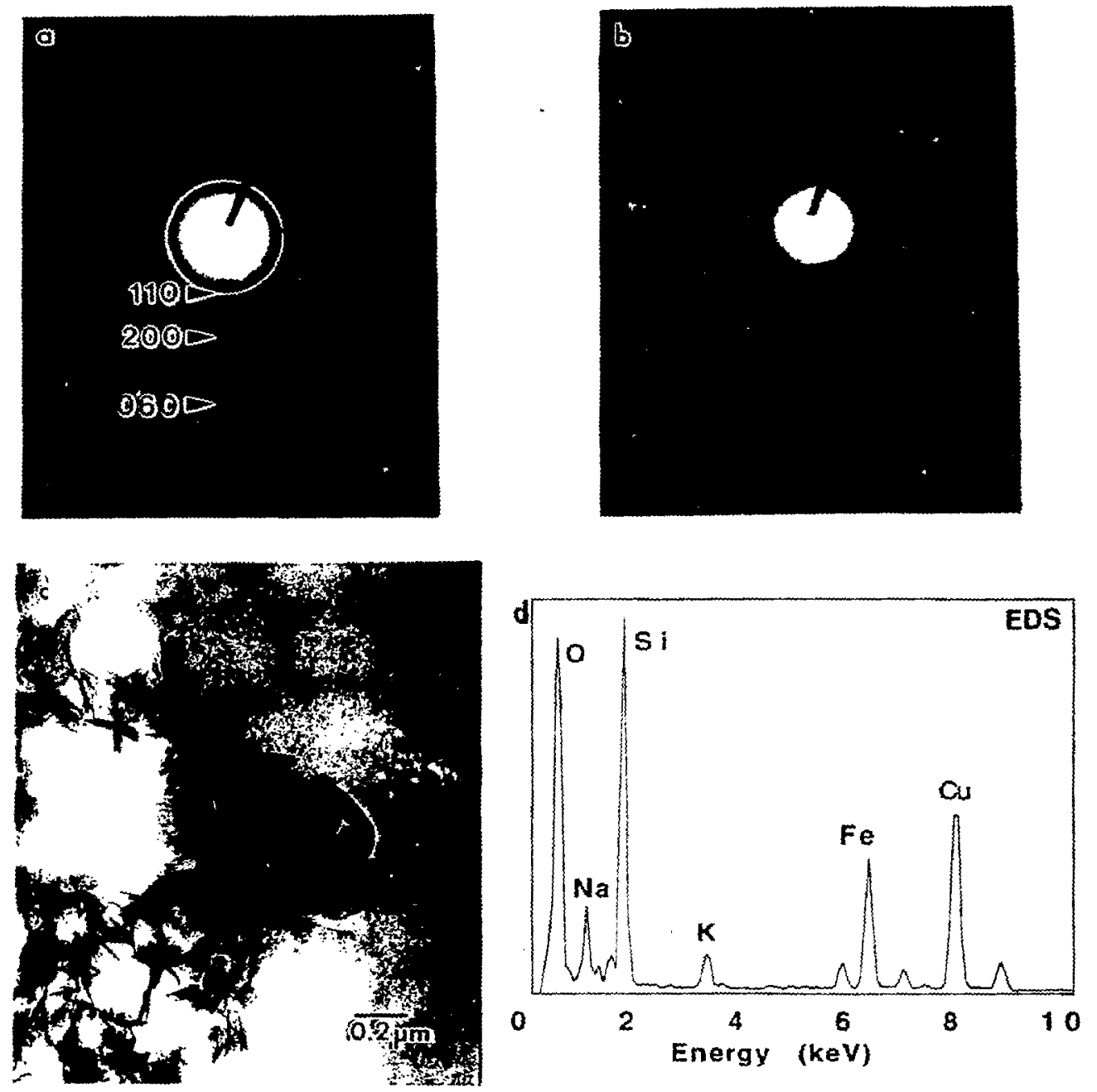
Fig. 4. (a) TEM image of a cross section through the surface layers of a reacted glass from the B-2 Test. showing attached uranium silicate phase, (b) micrograph of agglomerated particles from the B-2 test (Note attached clay material), (c) SAED pattern of the phase taken along the [010] zone axis and (d) EDS compositional analysis identify the phase as weeksite, a uranium silicate phase.
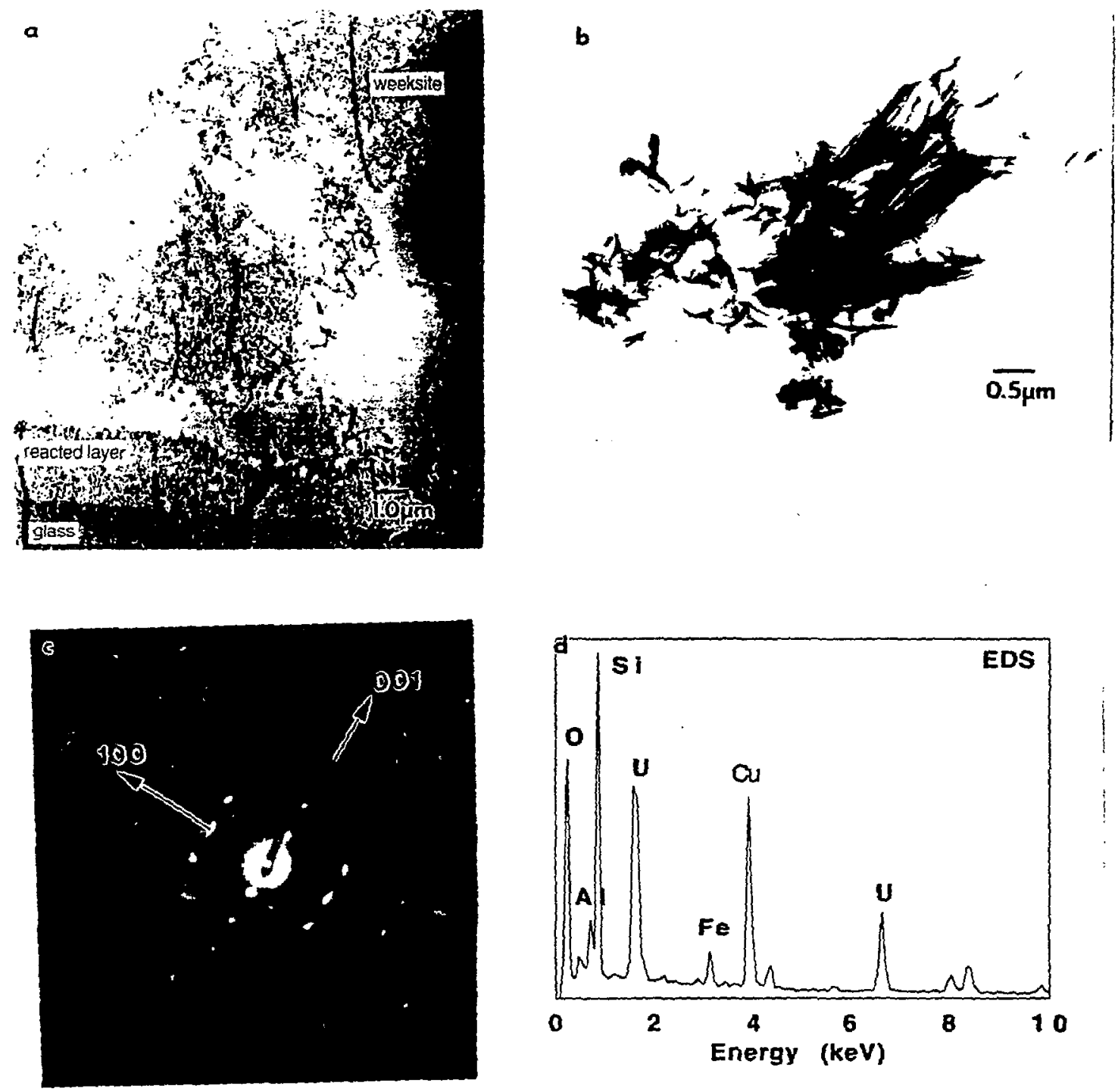


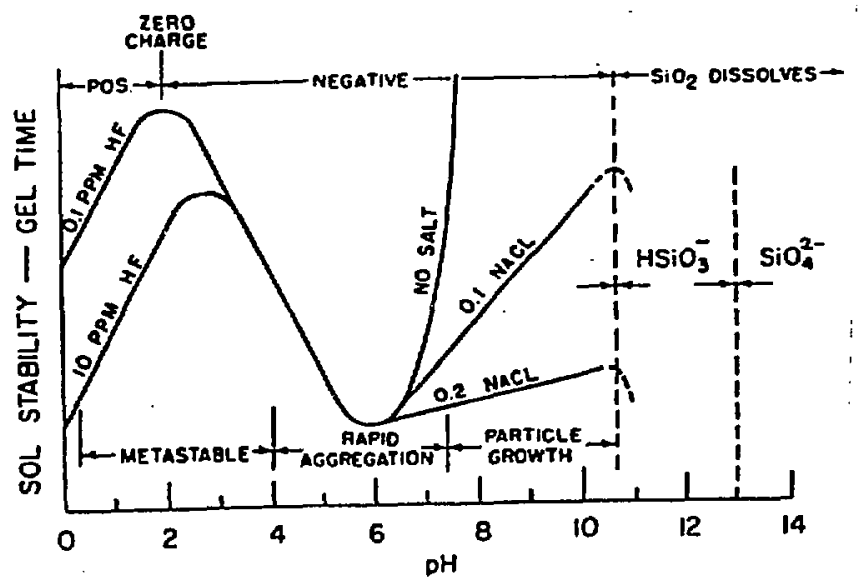

Fig. 5.

Effects of pH on Colloidal Silica-

Water System [23]. 